\title{
The effect of ex vivo lipopolysaccharide stimulation and nutrient availability on transition cow innate immune cell AKT/mTOR pathway responsiveness
}

\author{
Anja S. Sipka, ${ }^{1} \oplus$ Tawny L. Chandler, ${ }^{1} \odot$ Erica L. Behling-Kelly, ${ }^{1} \odot$ Thomas R. Overton, $^{2} \odot$ and Sabine Mann ${ }^{1 *}$ \\ ${ }^{1}$ Department of Population Medicine and Diagnostic Sciences, College of Veterinary Medicine, and \\ ${ }^{2}$ Department of Animal Science, College of Agriculture and Life Sciences, Cornell University, Ithaca, NY 14853
}

\begin{abstract}
Postpartum dairy cows experience a heightened inflammatory state coinciding with the time of greatest nutrient deficit. Nutrient availability is sensed on the cellular level by nutrient sensing kinases, such as the PI3K/AKT/mTOR (mTOR) pathway, a key orchestrator of immune cell activation and inflammatory balance. Our objective was to determine the responsiveness of this pathway to inflammatory stimulation with and without nutrient supplementation ex vivo. Blood samples were collected from Holstein cows (n $=14$ ) at $-42,-14,7,21$, and $42 \mathrm{~d}$ relative to calving. Control samples and samples pretreated with a mixture of amino acids, glucose, and insulin (AAM) were stimulated with $100 \mathrm{ng} / \mathrm{mL}$ E. coli lipopolysaccharide (LPS; LPS, AAMLPS) or left unstimulated (control, AAM). After $1 \mathrm{~h}$, ratios of mean fluorescence intensity for phosphorylated to total protein of AKT and mTORC1 substrates $\mathrm{S} 6 \mathrm{RP}$ and 4EBP1 were analyzed in polymorphonuclear cells (PMN), and monocytes by flow cytometry. A separate aliquot was stimulated with LPS for $2 \mathrm{~h}$ and relative mRNA abundance of $I L 10$, IL12A, IL12B, and TNFA in whole blood leukocytes from 10 cows was measured by reverse-transcription quantitative PCR. Repeated measures ANOVA was performed with fixed effects of time, treatment, and their interaction. Cells had different ratios of pathway proteins with PMN having the highest phosphorylation of AKT, S6RP, and 4EBP1. Stimulation with LPS consistently activated mTOR signaling in PMN regardless of nutrient supplementation except for postpartum $4 \mathrm{EBP} 1$, which increased in response to nutrients alone. In monocytes, AKT baseline phosphorylation was lower and activation could not be induced by either treatment, whereas activation of $4 \mathrm{EBP} 1$ responded to nutri-
\end{abstract}

Received July 22, 2019.

Accepted October 21, 2019

*Corresponding author: sm682@cornell.edu ent supplementation. Treatment with LPS increased phosphorylation of S6RP in both innate immune cell types. Nutrient supplementation increased baseline IL10 expression and decreased baseline as well as LPSinduced IL12B and TNFA expression. We conclude that the mTOR pathway in bovine innate immune cells can be differentially activated in response to inflammatory stimulation and nutrient supplementation in monocytes versus PMN. Effects of nutrient supplementation on cytokine mRNA abundance are likely specific to immune cell type.

Key words: inflammation, mTOR pathway, transition, amino acids

\section{INTRODUCTION}

Optimal management of transition cows has been the focus of a great number of studies in recent decades. Yet morbidity and mortality, particularly due to metabolic, inflammatory, and infectious disease, are still increased dramatically in the first $30 \mathrm{~d}$ after calving compared with all other stages of the production cycle (Alvåsen et al., 2014; Shahid et al., 2015). Postpartum cows experience a period of immune dysfunction characterized by heightened inflammatory response, greater susceptibility to infection, and decreased ability for efficient pathogen clearance, and this coincides with the nadir in nutrient and energy balance (Sordillo et al., 1995; Kimura et al., 1999; Hammon et al., 2006). Controlled inflammation is crucial for physiological functions associated with parturition and expulsion of the placenta, and a systemic inflammatory state is present in cows postpartum even when disease is not apparent (Bionaz et al., 2007). However, inflammation that is excessive in magnitude, duration, or both leads to host tissue injury, cell death, and inadequate pathogen clearance (Bauerfeld et al., 2012) and failure of resolution of inflammatory response plays a key role in immune dysfunction during the transition period (Bradford et al., 2015).

The central intracellular pathway that senses nutrient balance is the PI3K/AKT/mTORC1 kinase pathway 
(mammalian target of rapamycin, mTOR pathway), and it has emerged as a key orchestrator in integrating metabolic cues in immune cell activation in other mammalian species (Weichhart et al., 2015). The mTOR pathway senses the primary nutrients glucose and AA, as well as energy and oxygen availability, and maintains a critical balance between immune regulation and inflammation (Cobbold, 2013; Katholnig et al., 2013). This pathway is activated in immune cells by various extracellular signals, such as growth factors, cytokine binding, and toll-like receptor (TLR) ligands including LPS (Weichhart et al., 2015; Jones and Pearce, 2017). The mTOR pathway controls basic cellular processes such as translation and protein synthesis through its substrates [e.g., eIF4E-binding protein 1 (4EBP1) and S6 ribosomal protein $(\mathbf{S 6 R P})]$ and normally prevents exaggerated innate immune responses (Weichhart et al., 2008; Troutman et al., 2012; Vergadi et al., 2017). Depression of mTOR signaling in innate immune cells enhances and prolongs pro-inflammatory [e.g., IL-12, tumor necrosis factor (TNF)- $\alpha$, and IL-6] and blocks anti-inflammatory (IL-10) cytokine production following stimulation (Weichhart et al., 2008; Haidinger et al., 2010; Medina et al., 2010). We previously demonstrated AKT signaling in bovine adipose and muscle tissue decreases shortly after calving (Mann et al., 2016a,b). Given the relative deficit of glucose, AA, and energy postpartum, a role of the mTOR pathway in the observed immune dysfunction during this time is plausible. We recently explored mTOR signaling by immunoblotting in bovine peripheral blood mononuclear cells through the transition period and found that similar to other tissues the baseline AKT/mTOR pathway activation was decreased in immune cells postpartum (Mann et al., 2018), but responsiveness to stimulation was not tested. To the best of our knowledge, mTOR responsiveness of different immune cells to TLR stimulation and nutrient availability in transition dairy cows has not been described. Yet, the coinciding hallmarks of the transition period, nutrient deficit and heightened inflammatory state, warrant the investigation into this pathway in the dairy cow.

The main objective of this study was to assess response of the nutrient-sensing kinase pathway to LPS in different innate immune cell types with and without additional nutrients and insulin supplied ex vivo. The second objective was to describe associations of these treatments with cytokine mRNA abundance profiles. We hypothesized that responsiveness of the pathway is altered during the nutrient deficit of early lactation, that responsiveness depends on cell type, and that nutrient supplementation alters the inflammatory response.

\section{MATERIALS AND METHODS}

\section{Animals and Blood Samples}

Sampling. All procedures were evaluated and approved by the Cornell University Institutional Animal Care and Use Committee (protocol no. 2018-0023). Cows $(n=14)$ were sampled in this prospective cohort study from the Cornell University College of Veterinary Medicine Teaching dairy herd between August 2018 and February 2019. Inclusion criteria were absence of infectious diseases before dry-off, cows entering second or third lactation, absence of lameness, and having a predicted calving date between 28 and $42 \mathrm{~d}$ from the day of enrollment. Cows were housed in freestalls, milked 3 times per day, and fed once daily. All cows were fed the same dry cow and lactating ration, respectively. Recording and treatments for health events in all sampled cows were performed according to standard farm protocols.

Blood samples were obtained at the same time before the morning feeding for every sampling event (0700-0800 h) from the coccygeal vessels at wk 6 and 2 prepartum $(-6$ and $-2 \mathrm{wk})$ relative to expected calving, as well as 1,3 , and 6 wk postpartum $(+1,+3,+6$ wk) using a 20 $\mathrm{g} \times 2.5 \mathrm{~cm}$ needle after disinfection of the puncture site with $70 \%$ ethanol. Blood was first collected into $5-\mathrm{mL}$ sterile evacuated vacutainers with $0.05 \mathrm{~mL}$ of $15 \%$ EDTA (BD Vacutainer Systems, Franklin Lakes, NJ). Next, blood was collected into $10-\mathrm{mL}$ sterile evacuated glass tubes containing 143 USP units of freeze-dried sodium heparin (BD). To prevent contamination of the blood sample for assessment of inflammation and flow cytometry, these tubes were filled last. Samples were transported immediately to the laboratory.

Energy Metabolites. For assessment of plasma glucose, BHB, and nonesterified fatty acid (NEFA) concentrations, heparinized plasma was harvested from $10 \mathrm{~mL}$ of whole blood by centrifugation at 3,000 $\times g$ for $20 \mathrm{~min}$ at $4^{\circ} \mathrm{C}$. Samples were aliquoted and frozen at $-80^{\circ} \mathrm{C}$ until analysis. Samples were thawed at $4^{\circ} \mathrm{C}$ and all analyses were performed on the same day. Concentrations of glucose and NEFA were determined in triplicate using enzymatic colorimetric methods (PGO enzyme preparation, Sigma Aldrich, St. Louis, MO, and HR Series NEFA-HR (2), Wako Life Sciences, Mountain View, CA) as previously described (Mann et al., 2017). Plasma BHB concentrations were determined as a single measurement using the Precision Xtra point of care device (Abbott Diabetes Care, Abbott Park, IL) after heating plasma to $37^{\circ} \mathrm{C}$ for $10 \mathrm{~min}$ (Leal Yepes et al., 2018). In-house control samples of low and high concentrations were run with each glucose and NEFA assay. The average intraassay $\mathrm{CV}( \pm \mathrm{SD})$ for glucose 
and NEFA assays were $1.9 \%( \pm 0.01)$ and $2.5 \%( \pm 0.01)$, respectively.

Differential Blood Cell Counts and Packed Cell Volume. Blood smears were produced within 1 $\mathrm{h}$ of collection from EDTA blood, stained with WrightGiemsa using an automated stainer (Hematek, Siemens, Erlangen, Germany), and stored for later evaluation of all smears for red blood cell morphology and determination of total and differential white blood cell count by a board-certified clinical pathologist. Packed cell volume (PCV) was determined using microhematocrit tubes (Hemato-Clad Plain, Drummond Scientific, Broomall, PA).

Whole Blood Ex Vivo Treatments and LPS Stimulation. Heparinized whole blood samples were kept at room temperature and $1.9 \mathrm{~mL}$ was transferred into each of 4 sterile, endotoxin-free 5 -mL culture tubes (VWR, Radnor, PA). Two samples were treated with $100 \mu \mathrm{L}$ of an amino acid, glucose, and insulin mix (AAM; 10× non-EAA solution, 10× EAA solution, $10 \times$ insulin transferrin selenium mix, $2,000 \mathrm{mg} / \mathrm{dL}$ glucose in PBS, Thermo Fisher Scientific, Waltham, MA) to give a final supplementary concentration of AAM and insulin transferrin selenium in whole blood of $0.5 \times$ and $100 \mathrm{mg} / \mathrm{dL}$ added glucose, respectively. The final concentration of added nutrient components is summarized in Supplemental Table S1 (https:// doi.org/10.3168/jds.2019-17307). The 2 remaining vials received $100 \mu \mathrm{L}$ of PBS as control (CTRL). All samples were then incubated for $1 \mathrm{~h}$ at $37^{\circ} \mathrm{C}$ under agitation. After $1 \mathrm{~h}, 1$ vial of AAM and 1 vial of CTRL were each stimulated with $20 \mu \mathrm{L}$ of a $10 \mu \mathrm{g} / \mathrm{mL}$ LPS solution (Escherichia coli O111:B4, Millipore Sigma, Burlington, MA) to achieve a final LPS concentration of $100 \mathrm{ng} / \mathrm{mL}$, and the remaining 1 vial of CTRL and AAM treatments received $20 \mu \mathrm{L}$ of PBS. The resulting 4 treatments of this experimental set-up were CTRL, LPS, AAM, and AAMLPS. All treatments were incubated at $37^{\circ} \mathrm{C}$ under agitation in an incubating orbital shaker (VWR, Radnor, PA).

\section{Flow Cytometry}

One hour after addition of LPS or PBS, whole blood in all treatments was lysed, fixed, and permeabilized using the Phosflow lyse-fix and perm buffer system (BD Biosciences, San Jose, CA). Briefly, lysis of red blood cells and fixation of whole blood leukocytes was performed with Lyse/Fix buffer I (BD) at $37^{\circ} \mathrm{C}$ for $10 \mathrm{~min}$. Subsequently samples were centrifuged for 8 min at $500 \times g$ and washed one more time with PBS for $10 \mathrm{~min}$ at $250 \times \mathrm{g}$. Cell pellets were then placed on ice and permeabilized by adding Perm buffer III (BD) while vortexing and incubated for $30 \mathrm{~min}$ on ice.
After incubation, cells were washed twice with PBS for $10 \mathrm{~min}$, centrifuged at $250 \times \mathrm{g}$, and cooled to $4^{\circ} \mathrm{C}$ until antibody labeling. In samples from 6 cows representing all treatment conditions, aliquots from each treatment were lysed but not fixed to test viability of the cells. After lysis of erythrocytes, cell pellets were resuspended in PBS containing propidium iodide at a final concentration of $2 \mu \mathrm{g} / \mathrm{mL}$. Cells were analyzed by flow cytometry immediately upon staining.

Antibody Labeling. Cell pellets from each of the 4 treatments per cow and time point were split into 3 aliquots. Each aliquot was labeled with an antibody to detect surface CD14 (Bio-Rad Laboratories, Hercules, CA) and an antibody pair to detect intracellular expression of phosphorylated (p) and total AKT, pS6RP and S6RP, or p4EBP1 and 4EBP1 (Cell Signaling Technology, Danvers, MA; Table 1). Antibodies were validated as cross-reactive for bovine species by the manufacturer (CD14, pAKT) or documented in a previous study to be cross-reactive for bovine species (Mann et al., 2018). Additionally, we performed Western blots on mouse liver samples as positive controls and 2 separate bovine immune cell lysates for every monoclonal antibody according to a technique previously described (Mann et al., 2018). For flow cytometry, samples were incubated for 30 min at room temperature with antibodies for the respective phosphorylated and total protein target, as well as CD14 (final antibody concentration, see Table 1). All used isotypes were tested for unspecific binding to bovine blood leukocytes, using isotype controls for mouse $\operatorname{IgG}_{1}$ (Bio-Rad Laboratories, Hercules, CA) and rabbit IgG (Cell Signaling Technology) at the same final concentration as specific antibodies. Cells were subsequently washed twice and resuspended in PBS for flow cytometry analysis.

Flow Cytometry Analysis. All samples were measured in an Attune acoustic focusing flow cytometer (Thermo Fisher Scientific, Waltham, MA) equipped with a blue (BL, $488 \mathrm{~nm}$ ) and a violet laser (VL, 405 $\mathrm{nm}$ ) for fluorescence detection. Leukocytes were gated based on morphology in forward and side scatter and 20,000 events in the leukocyte gate were acquired per measurement. All data were analyzed in FlowJo V10 software (BD Biosciences, San Jose, CA). Gating was used to distinguish PMN (all granulocytes) from mononuclear cells (monocytes and lymphocytes; see Figure 1). Expression of surface CD14 was evaluated by fluorescence in channel VL-1 (Pacific Blue). Mean fluorescence intensity (MFI) for fluorescence channels BL-1 (ALEXA 488), capturing intracellular expression of phosphorylated protein, and BL-2 (Phycoerythrin), capturing intracellular expression of total protein for PMN and $\mathrm{CD}_{14}{ }^{+}$monocytes, were recorded for all samples (Figure 1). For analysis of viability, cells were 
Table 1. Antibodies used for surface and intracellular labeling of bovine blood leukocytes ${ }^{1}$

\begin{tabular}{lllc}
\hline Antibody target (clone) & Source & Phosphorylation site & Conjugation \\
\hline CD14 (TÜK 4) & Mouse monoclonal IgG1 & Dilution \\
pAKT (D9E) & Rabbit monoclonal IgG & Ser473 & Pacific blue \\
AKT (C67E7) & Rabbit monoclonal IgG & Ser235/236 & ALEXA488 \\
pS6RP (D57.2.2E) & Rabbit monoclonal IgG & - & Phycoerythrin \\
S6RP (54D2) & Mouse monoclonal IgG1 & ALEXA488 \\
p4EBP1 (236B4) & Rabbit monoclonal IgG & - & $1: 25$ \\
4EBP1 (53H11) & Rabbit monoclonal IgG & Phycoerythrin & $1: 25$ \\
\hline
\end{tabular}

${ }^{1} \mathrm{p}$ = phosphorylated; antibodies purchased from Cell Signaling Technology (Danvers, MA) except for CD14, which was purchased from Bio-Rad AbD Serotec (Hercules, CA).

gated by morphology and dead cells were identified as propidium iodide positive events in unfixed samples in fluorescence channel BL-3.

\section{PCR Analysis}

Treatments and RNA Extraction. In a parallel set of incubations, $2 \mathrm{~h}$ after addition of LPS or PBS, the reaction was stopped on ice, followed by centrifugation at $1,500 \times g$ at $4^{\circ} \mathrm{C}$ for $15 \mathrm{~min}$. Plasma was separated and stored separately. Cell pellets were prepared as previously described (Mann et al., 2018). Briefly, red blood cells in the cell pellet were lysed using ice-cold aluminum-chloride-potassium red cell lysis buffer (ACK buffer, Lonza, Basel, Switzerland). Cells were pelleted at $300 \times g$ for $5 \mathrm{~min}$ at $4^{\circ} \mathrm{C}$ and washed twice with ice-cold PBS. The white blood cell pellet was lysed in RNA lysis buffer (RLT buffer, Qiagen, Hilden, Germany) with the addition of $10 \mu \mathrm{L} / \mathrm{mL} \beta$-mercaptoethanol (Sigma-Aldrich, St. Louis, MO) and stored at $-80^{\circ} \mathrm{C}$ until subsequent RNA extraction and characterization. The lysate was rapidly thawed at $37^{\circ} \mathrm{C}$ for $10 \mathrm{~min}$ and homogenized using a spin column (QIAshredder, Qiagen) before RNA was extracted using the RNeasy Plus Mini Kit (Qiagen). Extracted RNA was characterized and quantified using a NanoDrop One spectrophotometer (Thermo Fisher Scientific, Waltham, MA) with all samples having an optical density 260:280 ratio $\geq 1.9$ but $\leq 2.1$. To further describe RNA integrity, a set of 20 samples that equally represented all cows, sampling time point, and treatments were analyzed with a fragment analyzer for RNA quality control (4200 TapeStation System, Agilent Technologies, Santa Clara, CA) with a mean RNA integrity number of 9.5 and a range of 9.1 to 9.7 .

Reverse-Transcription Quantitative PCR. A volume containing $0.5 \mu \mathrm{g}$ of RNA was reverse transcribed (SuperScript IV First-Strand cDNA Synthesis, Life Technologies, Carlsbad, CA) and stored at $-20^{\circ} \mathrm{C}$. A pool of unstimulated and LPS-stimulated sample cDNA served as the calibrator sample. Bovine-specific primer probe sets (TaqMan Gene Expression Assays, Ap- plied BioSystems, Thermo Fisher Scientific) with exon spanning probes were purchased for genes of interest [IL12A (p35): Bt03213922, IL12B (p40): Bt03213923, TNFA: Bt03259154, IL10: Bt03212724] and 2 reference genes (RPLP0: Bt03218087, TBP: Bt03241946). Both reference genes were previously shown to be stably expressed in bovine leukocytes (Brym et al., 2013) and used in a similar experimental setup (Mann et al., 2018). Real-time reverse-transcription quantitative PCR was performed in triplicate using a 1:3 or 1:6 dilution of cDNA at $20 \%$ of the final $10-\mu \mathrm{L}$ reaction volume using TaqMan Gene Expression Master Mix (Applied BioSystems, Thermo Fisher Scientific) in a QuantStudio 12K Flex Real-Time PCR System (Applied BioSystems, Thermo Fisher Scientific). Run conditions for all genes of interest were 1 cycle at $50^{\circ} \mathrm{C}$ for $2 \mathrm{~min}, 1$ cycle at $95^{\circ} \mathrm{C}$ for $10 \mathrm{~min}$, followed by 40 cycles of $95^{\circ} \mathrm{C}$ for 15 $\mathrm{s}$ and $60^{\circ} \mathrm{C}$ for $1 \mathrm{~min}$. Raw data were analyzed using the comparative quantification method $(\Delta \Delta \mathrm{Ct}$ method, QuantStudio 12K Flex Software, v1.2.2) and expressed as relative quantity $\left(\mathrm{RQ}=2^{-\Delta \Delta \mathrm{Ct}}\right)$ before export for statistical analysis. The PCR analysis was completed on all samples from 10 animals with complete sample sets.

\section{Analytical Approach}

Repeated measures analysis of single outcomes per time point (energy metabolites, differential cell counts, PCV) was performed in Proc MIXED with the fixed effect of time and repeated statement of time point and subject of cow (random effect). $P$-values of pairwise comparisons of least squares means for the effect of time were adjusted for multiple comparisons using Tukey's posthoc procedure. For flow cytometry data, changes in the ratio of MFI for phosphorylated to total protein from CTRL (= baseline) were analyzed and the difference from 0 statistically analyzed as the null hypothesis.

Repeated measures of outcomes with several observations for each time point (PCR and flow cytometry data) were analyzed using Proc MIXED as described 
above with the fixed effects of time, treatment, and their interaction. $P$-values of pairwise comparisons of least squares means for the interaction of time and treatment were adjusted for multiple comparisons using Tukey's posthoc procedure. When the $F$-test for the interaction of time and treatment exceeded 0.05 , differences among the main effects of time and treatments were analyzed using Tukey's posthoc procedure on LSM for each main effect, respectively.

Outlier diagnostics were performed as defined a priori with the INFLUENCE statement and removed if Cook's distance exceeded 0.5. After each model fit, residuals were visually inspected for normality and homoscedasticity. The PCR data were log-transformed before analysis to fulfill model assumption of homoscedasticity and normality of residuals. All results are presented as least squares means and standard error except for PCR data, which is presented as geometric mean and backtransformed 95\% CI.

Differences in cell viability between treatments were analyzed using Kruskal-Wallis in JMP (v.11.0, SAS Institute Inc., Cary, NC).
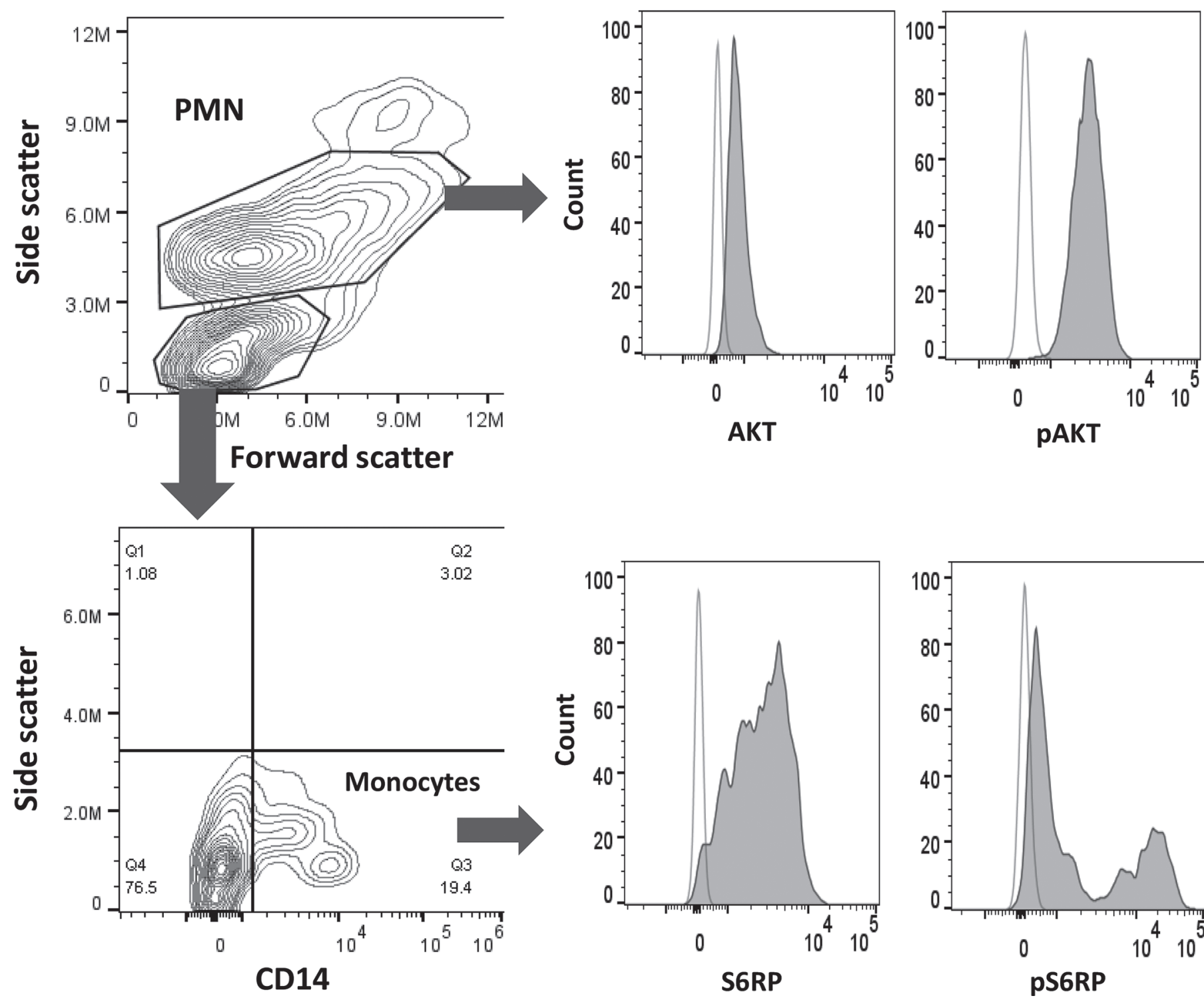

Figure 1. Gating strategy for whole blood leukocytes. Polymorphonuclear cells were distinguished by forward and side scatter. Monocytes were identified among mononuclear cells based on CD14 expression. Cells were intracellularly labeled with fluorochrome coupled antibodies against phosphorylated (p) and total protein, and mean fluorescence intensity was recorded. Contour plots and histograms represent examples from the analyzed data set. Solid fill histograms are samples labeled with antibodies against AKT, pAKT (PMN), S6RP, or pS6RP (CD14 monocytes), and clear histograms represent unlabeled controls. 


\section{RESULTS}

\section{Description of Study Population}

All animals were entering their second $(\mathrm{n}=13)$ or third $(\mathrm{n}=1)$ lactation and were dry for an average (SD) of 46 (12) d. Sampling occurred on d -37 (12), -12 (4), 7 (2), 21 (2), and 41 (3) relative to calving for the $-6,-2,+1,+3$, and +6 wk time points. Cows produced an average $39.5(9.9) \mathrm{kg}$ of milk at the first test day $(17 \pm 9$ DIM). Two cows had health events recorded during the study: one cow was treated for ketosis $(300 \mathrm{~mL}$ of propylene glycol orally once a day for $3 \mathrm{~d}$ ), and one developed metritis following retained placenta and twin calving. Both cows remained in the analysis. Three cows were missing one sample each $(-2$ wk) due to unexpected early calving.

\section{Energy Metabolites and Differential Blood Cell Counts}

Plasma concentrations of glucose, NEFA, and BHB are shown in Figure 2. Glucose concentrations decreased postpartum compared with prepartum concentrations, whereas NEFA and BHB concentrations increased postpartum. Packed cell volume decreased slightly throughout the study period and was lowest in +6 wk compared with -6 wk (Figure $3 \mathrm{~A}$ ). Monocyte counts, $\%$ of leukocytes, did not vary significantly over time $(P=0.65)$, whereas lymphocyte percentage reached a nadir in +1 wk (Figure $3 \mathrm{C}$ ) and segmented neutrophils reached their maximum counts at this time point (Figure 3D).

\section{Phosphorylation of mTOR Pathway Kinases in Response to AA and LPS}

Viability of Cells After Treatment and Stimulation. Viability for all tested samples was greater than $90 \%$ and treatment had no effect on viability $(P$ $=0.99)$ with median (range) viability of 96.9 (92.0 to 97.6), 96.8 (93.1 to 98), 96.4 (92.1 to 97.8), and 96.6 (90.5 to 97.9) in CTRL, AAM, LPS, and AAMLPS treatments, respectively.

$A A M / L P S$-Induced Change from Baseline. The ratio of phosphorylated to total protein MFI from intracellular flow cytometry calculated as change from baseline (CTRL) for the different immune cell subsets is shown in Figure 4. No interaction was detected for time and treatment for any of the subsets and protein targets. Differences over time and between treatments are therefore discussed separately below for each pathway protein and cell subset.

$p A K T: A K T$ Ratio. An effect of treatment was found in PMN such that the pAKT:AKT ratio in AAM was lower than AAMLPS $(-0.12 \pm 0.03 ; P=0.001)$, AAM was lower than LPS $(-0.13 \pm 0.03 ; P=0.001)$, but AAMLPS was not different from LPS $(-0.002 \pm$ $0.03 ; P=0.99)$. For time point comparisons only +1 wk was lower than +6 wk $(-0.12 \pm 0.04 ; P=0.03)$.

In monocytes, AAMLPS led to a higher ratio of pAKT:AKT compared with LPS alone $(0.10 \pm 0.04 ; P$ $=0.06)$, but other treatments did not differ from each other $(P \geq 0.40)$. Time had an effect on the pAKT: AKT ratios such that compared with -2 wk they were lower in $+1 \mathrm{wk}(-0.12 \pm 0.04 ; P=0.02),+3 \mathrm{wk}(-0.15$ $\pm 0.04 ; P=0.004)$, and +6 wk $(-0.15 \pm 0.04 ; P=$ $0.004)$.

pS6RP:S6RP Ratio. An effect of time and treatment was found for all cell subsets. For treatments in PMN, the ratio was lower in AAM than AAMLPS $(-0.48 \pm 0.05 ; P=0.001)$, lower in AAM than LPS $(-0.36 \pm 0.05 ; P=0.001)$, and in addition AAMLPS led to a higher ratio than LPS alone $(0.12 \pm 0.05 ; P$ $=0.04)$. Time points differing in pS6RP:S6RP ratio in PMN were -2 wk was lower than +3 wk $(-0.38 \pm$ $0.07 ; P<0.001),+1$ wk was lower than +3 wk $(-0.28$
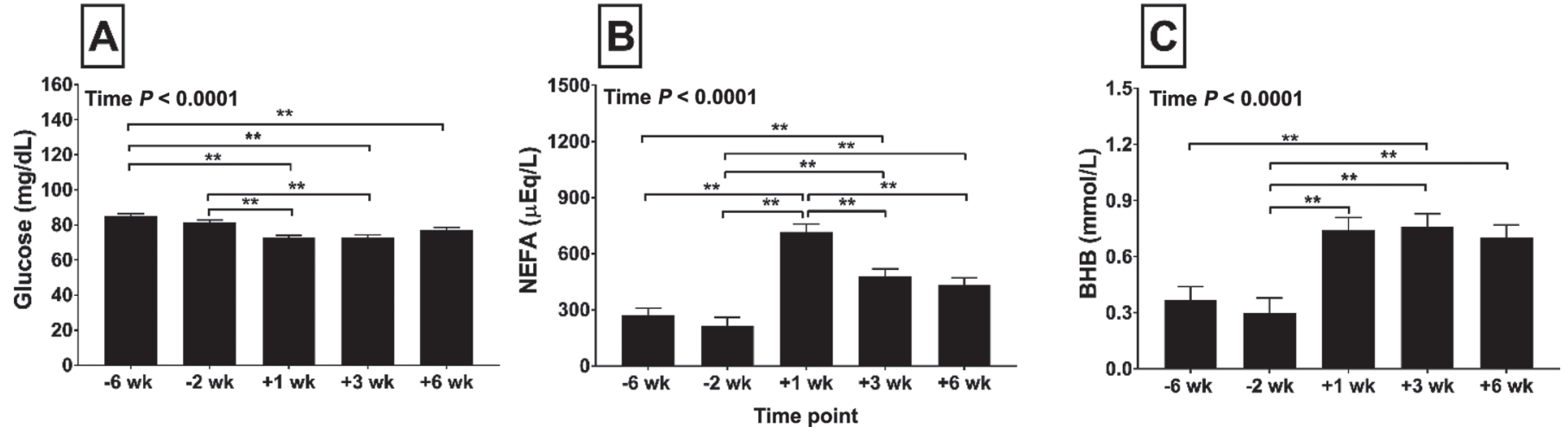

Figure 2. Least squares means $( \pm \mathrm{SE})$ of plasma glucose $(\mathrm{mg} / \mathrm{dL} ; \mathrm{A})$, nonesterified fatty acids $(\mathrm{NEFA} ; \mu \mathrm{Eq} / \mathrm{L} ; \mathrm{B})$, and BHB $(\mathrm{mmol} / \mathrm{L} ; \mathrm{C})$ concentrations at each sampling time point for all cows $(\mathrm{n}=14)$. Pairwise comparisons of time points marked $* *$ differ at $P<0.001$ in repeated measures ANOVA with Tukey's posthoc procedure. 
$\pm 0.06 ; P=0.001),+3$ wk was higher than $-4 \mathrm{wk}(0.27$ $\pm 0.06 ; P=0.002)$, and -2 wk was lower than +6 wk $(-0.19 \pm 0.07 ; P=0.04)$.

In monocytes, the pS6RP:S6RP ratio was lower in AAM than in AAMLPS $(-0.29 \pm 0.07 ; P<0.001)$ and AAM was lower than LPS $(-0.18 \pm 0.07 ; P=0.02)$, but AAMLPS was not different from LPS alone $(0.11 \pm$ 0.07; $P=0.26$ ). Time differences were found such that the ratio of pS6RP:S6RP was lower in +1 wk compared with -2 wk $(-0.19 \pm 0.07 ; P=0.05)$ and compared with +3 wk $(-0.22 \pm 0.06 ; P=0.004)$.

p4EBP1:4EBP1 Ratio. An effect of time and treatment was found for PMN. The ratio was lower for AAM compared with AAMLPS $(-0.25 \pm 0.06 ; P$ $=0.001)$, AAMLPS had a higher ratio than LPS (0.13 $\pm 0.06 ; P=0.08)$, but AAM did not differ from LPS $(-0.11 \pm 0.06 ; P=0.20)$. When comparing different time points, $+1 \mathrm{wk}$ was higher than $-6 \mathrm{wk}(0.25 \pm$ $0.08 ; P=0.01),+3 \mathrm{wk}$ was higher than -2 and $-6 \mathrm{wk}$ $(0.32 \pm 0.08$ and $0.40 \pm 0.08$, respectively; $P<0.01)$, and +6 wk was higher than -2 and -6 wk $(0.31 \pm 0.08$ and $0.38 \pm 0.08$, respectively; $P<0.001$ ).

In monocytes, AAM had a greater ratio of p4EBP1: 4EBP1 than LPS $(0.19 \pm 0.04 ; P<0.001)$ and AAMLPS had a greater ratio than LPS $(0.17 \pm 0.04 ; P$ $=0.001)$, but AAM did not differ from AAMLPS (0.02 $\pm 0.04 ; P=0.86)$. None of the time points differed from each other.

Baseline Phosphorylation Comparison of Cell Subsets. Figure 5 shows the comparison of phosphory-
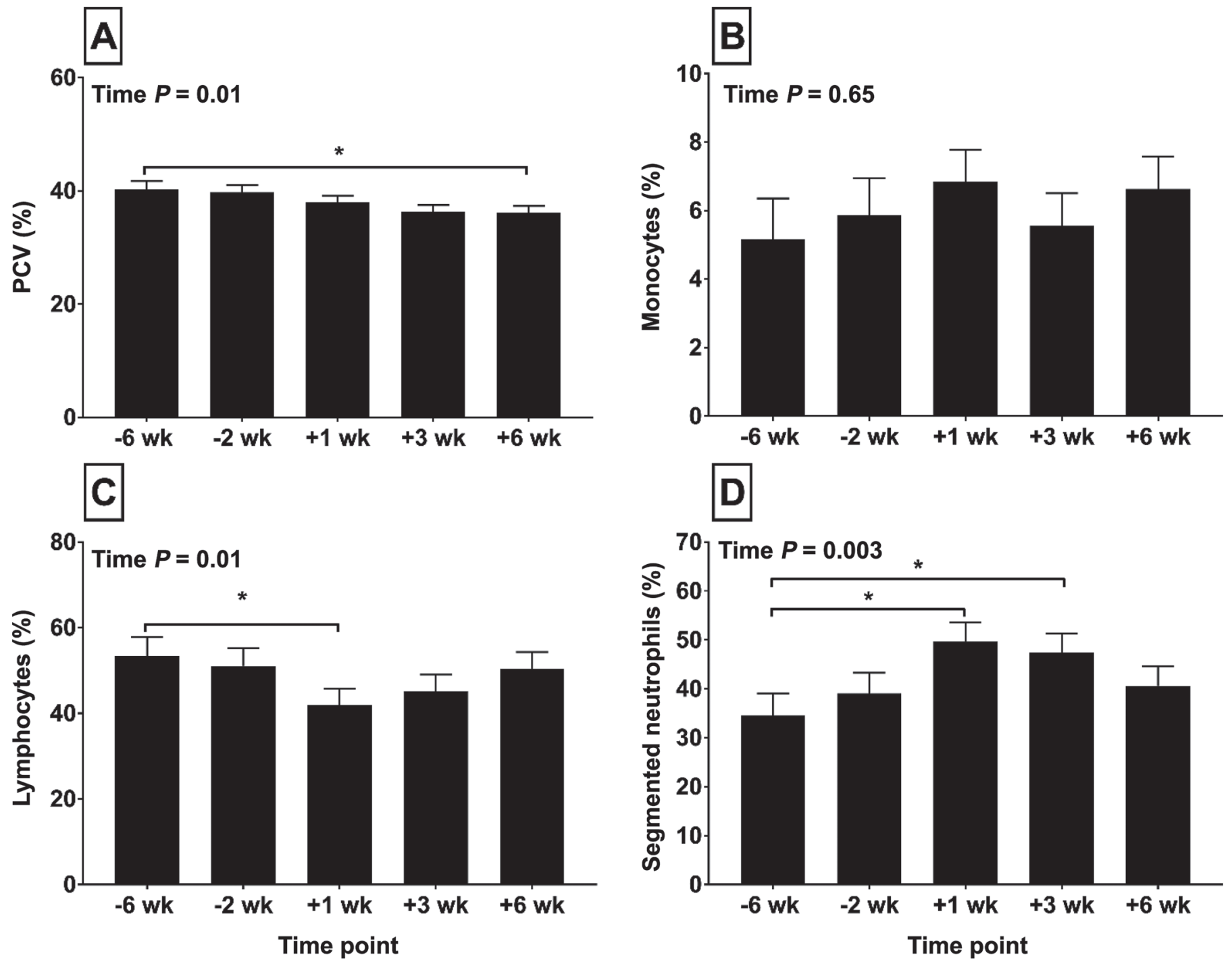

Figure 3. Least squares means ( \pm SE) of EDTA blood packed cell volume (PCV, \%; A), monocytes (\% of leukocytes; B), lymphocytes (\% of

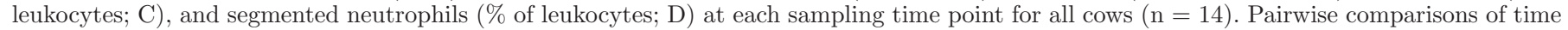
points marked with $*$ differ at $P<0.05$ in repeated measures ANOVA with Tukey's posthoc procedure. 
Polymorphonuclear cells
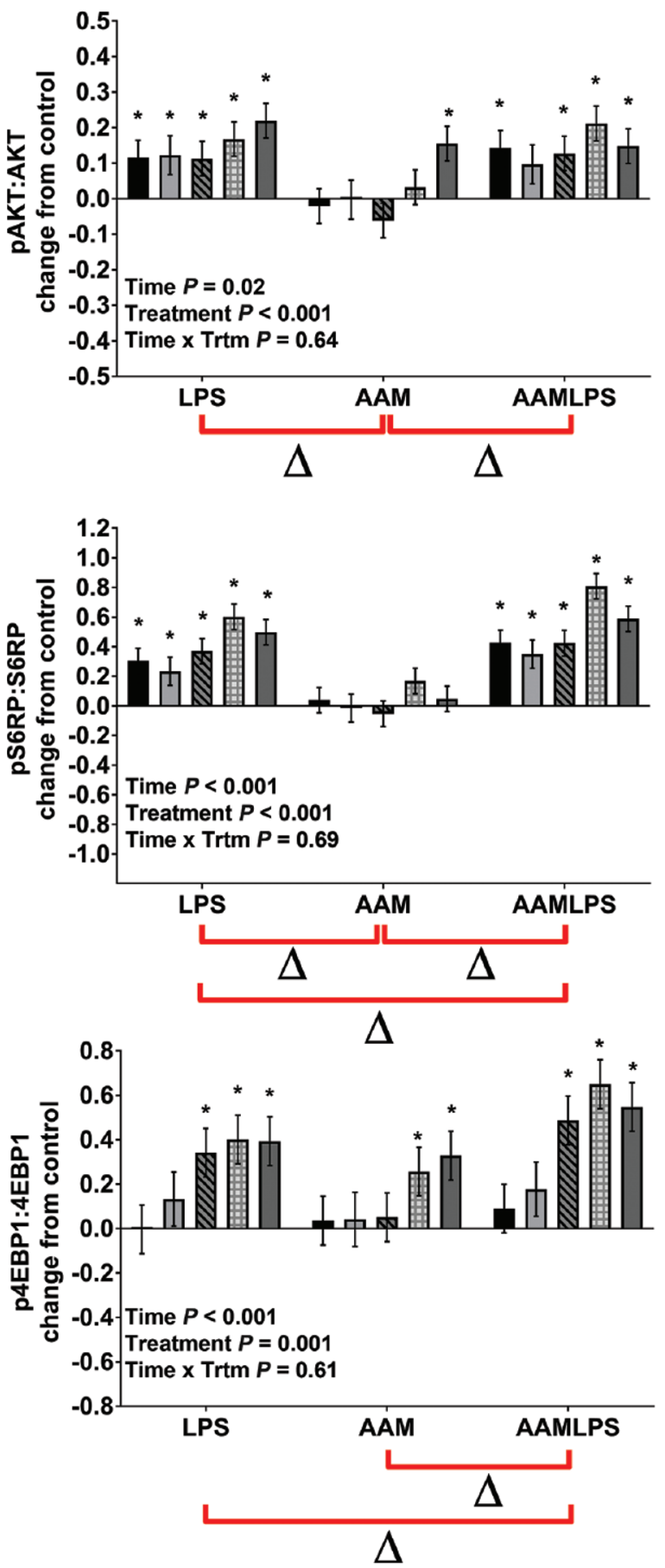

Monocytes
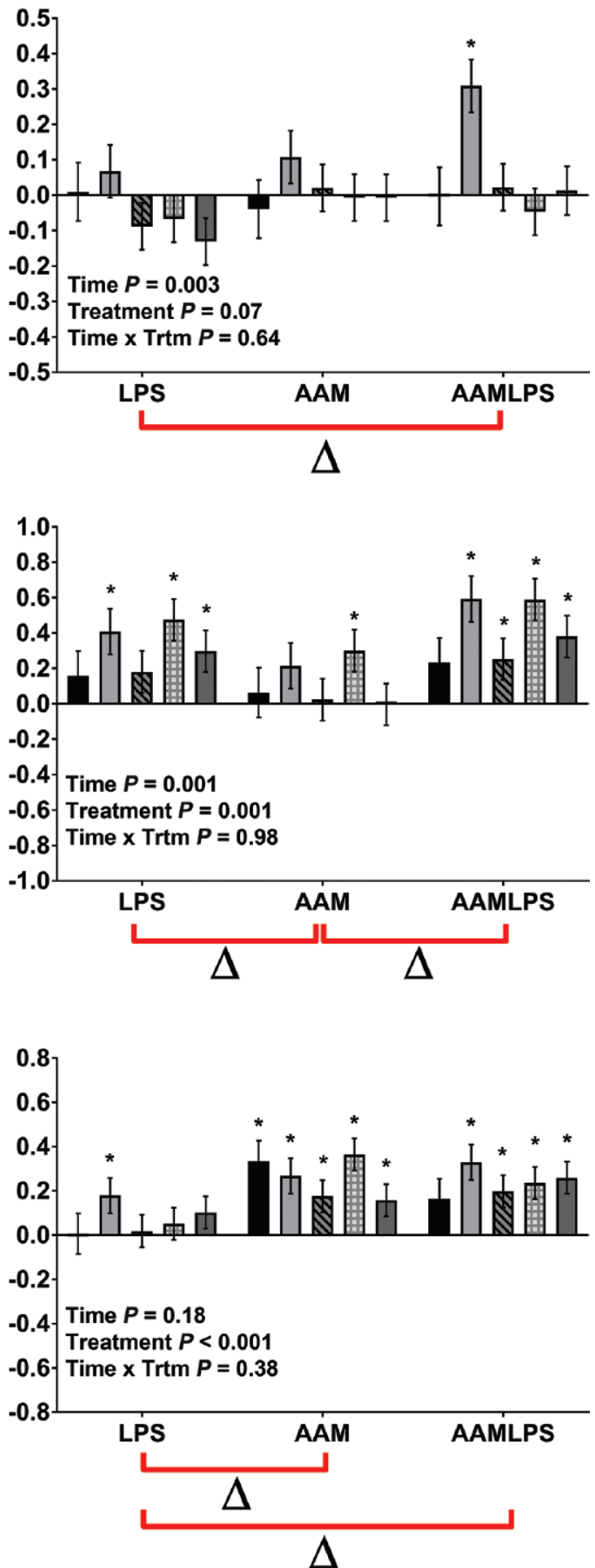

\section{$\square-6$ wk $\square-2$ wk $\triangle+1$ wk $⿴+3$ wk $\square+6$ wk}

Figure 4. Least squares means $( \pm \mathrm{SE})$ of the ratio of phosphorylated (p) to total protein of interest in PMN (left) and monocytes (right) of cows $(\mathrm{n}=14)$ expressed as relative change to untreated whole blood. Top row: pAKT (Ser473):AKT; middle row pS6RP (Ser235/236):total S6RP; bottom row p4EBP1 (Thr37/46):total 4EBP1. Whole blood was incubated for $1 \mathrm{~h}$ with PBS as control (CTRL) or $0.5 \times$ of an amino acid/ insulin/glucose mix diluted in PBS (AAM). Whole blood was then stimulated with $100 \mathrm{ng} / \mathrm{mL}$ of Escherichia coli LPS for $1 \mathrm{~h}$ (LPS; AAMLPS), or remained unstimulated (AAM). All results are presented as change from control. Ratio of p:total protein was determined by intracellular flow cytometry. $P$-values for fixed effects of time, treatment (Trt), and their interaction extracted from repeated measures ANOVA; $*$ denotes difference from 0 (CTRL) at $P<0.05$; treatments marked with $\Delta$ differed at $P<0.05$ in Tukey's posthoc test. 


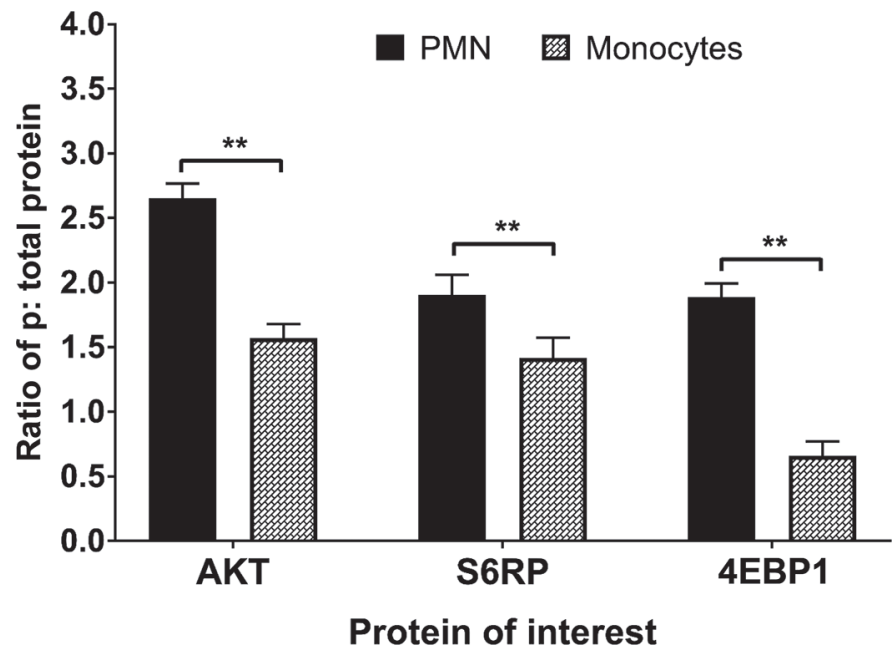

Figure 5. Least squares means $( \pm \mathrm{SE})$ of the ratio of phosphorylated (p) to total protein mean fluorescence intensity for each protein of interest (4EBP1, AKT, S6RP1) in PMN and monocytes across all sampling time points in unstimulated control samples: pAKT (Ser473):AKT; pS6RP (Ser235/236):total S6RP, p4EBP1 (Thr37/46):total 4EBP1. Ratio of phosphorylation was determined by intracellular flow cytometry. Repeated measures ANOVA accounting for repeated effect of time; LSM of main effect of cell type is presented. Pairwise comparisons marked with ${ }^{* *}$ reached $P<0.001$.

lation ratios between cell subsets across all time points in unstimulated CTRL cells. At baseline, PMN had higher ratios for all proteins of interest compared with monocytes $(P<0.001)$.

\section{Total Leukocyte Cytokine mRNA Abundance}

The geometric mean and back-transformed CI of the expression of all genes of interest without LPS addition (baseline) and with LPS stimulation are depicted in Figure 6. In the absence of an interaction effect, pairwise comparisons of time points and treatment differences were explored for baseline and stimulated mRNA abundance, respectively.

Baseline Control. Log mRNA abundance of IL10 was higher in AAM than in CTRL $(0.23 \pm 0.12 ; P$ $=0.05)$. Relative log mRNA abundance was higher at +1 wk than at -2 and -6 wk $(0.71 \pm 0.19$ and 0.83 \pm 0.19 , respectively; $P<0.01$ ), higher at +3 wk than at -6 wk $(0.52 \pm 0.18 ; P=0.05)$, and higher at +6 wk than at -6 wk $(0.58 \pm 0.18 ; P=0.02)$. No differences between treatments or time points were found for IL12A at baseline.

Supplementation of AAM decreased log IL12B mRNA abundance at baseline $(-0.23 \pm 0.12 ; P=$ 0.06). Abundance of mRNA increased at +1 wk compared with -2 and $-6 \mathrm{wk}(1.10 \pm 0.19$ and $1.21 \pm 0.19$, respectively; $P<0.001$ ), at +3 wk compared with -2 and -6 wk $(0.67 \pm 0.19$ and $0.78 \pm 0.19$, respectively;
$P=0.01$ ), and at $+6 \mathrm{wk}$ compared with -2 and $-6 \mathrm{wk}$ $(0.63 \pm 0.20$ and $0.73 \pm 0.19$, respectively; $P<0.02)$.

Supplementation of AAM decreased log TNFA mRNA abundance at baseline $(-0.29 \pm 0.15 ; P=$ $0.05)$. Abundance of mRNA was increased at $+1 \mathrm{wk}$ compared with -2 and -6 wk $(0.95 \pm 0.23$ and $0.82 \pm$ $0.22 ; P<0.01)$. Abundance of mRNA was higher at +3 and +6 wk compared with -2 wk $(0.60 \pm 0.23$ and 0.73 \pm 0.22 , respectively; $P<0.09$ ), and mRNA abundance was higher at +6 wk compared with -6 wk $(0.60 \pm$ $0.22 ; P=0.07)$.

LPS Stimulated. Stimulated log mRNA abundance differed from baseline for all genes of interest with mRNA abundance increasing for all targets $(P<$ 0.001). Stimulated log mRNA abundance of $I L 10$ and IL12A were not different between AAMLPS and LPS or between any of the time points.

Stimulated log mRNA abundance of IL12B was lower in AAMLPS than in LPS $(-1.02 \pm 0.14 ; P<0.001)$. Log mRNA abundance was lower at -2 and +3 wk compared with -6 wk $(-0.60 \pm 0.22$ and $-0.78 \pm 0.22$, respectively; $P<0.06)$.

Stimulated $\log$ mRNA abundance of TNFA was lower in AAMLPS than in LPS $(-0.62 \pm 0.12 ; P<0.001)$, and did not differ over time.

\section{DISCUSSION}

The main objective of this study was to assess the LPS responsiveness of the nutrient-sensing kinase pathway in different immune cell subsets with and without additional nutrients supplied ex vivo.

We found that LPS differentially activates pathways in bovine immune cell subsets. In PMN, phosphorylation of all investigated proteins of the mTOR pathway increased continuously from $6 \mathrm{wk}$ prepartum to $6 \mathrm{wk}$ postpartum in response to LPS. In neutrophils, the activation of mTOR is an important mechanism for a rapid response to inflammatory signals and has been described in mice as a predominantly pro-inflammatory pathway (Weichhart et al., 2015; Zhao et al., 2017). Less is known about mTOR signaling in other granulocytes such as eosinophils and basophils (Weichhart et al., 2015) that account for a small percentage of circulating granulocytes. The continuous increase of mTOR responsiveness in PMN postpartum in response to LPS could be an indication for a more pronounced proinflammatory activity in this cell population in early lactation. In PMN, we did not detect a change in AKT responsiveness with additional nutrients, but nutrient supplementation did increase LPS-induced phosphorylation of mTOR substrates S6RP and 4EBP1. The mTORC1 kinase specifically senses AA availability and regulates downstream substrates as a result, whereas 


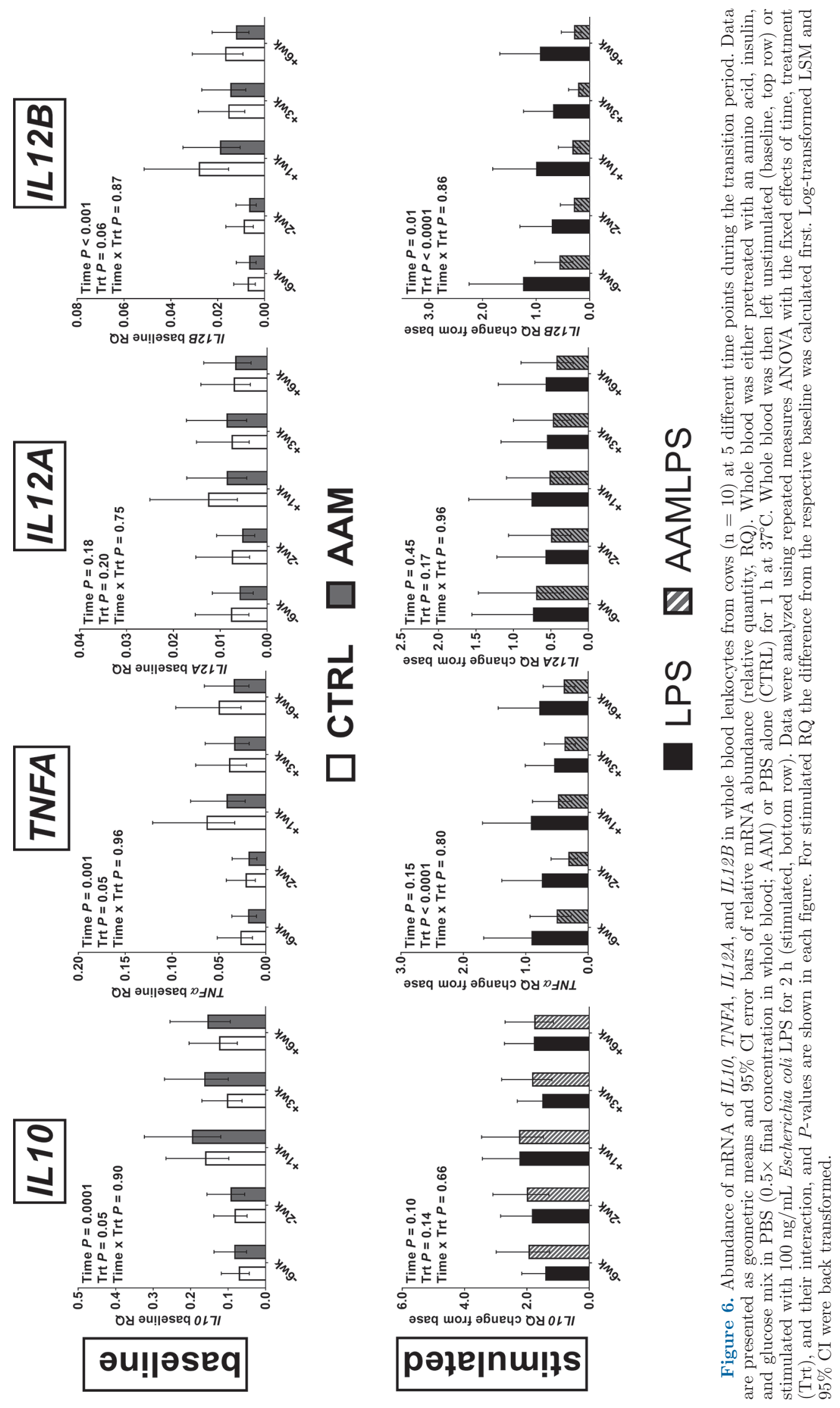


AKT phosphorylation follows insulin signaling upstream of mTORC1 (Laplante and Sabatini, 2009).

Circulating PMN are terminally differentiated effector cells with a short life span of approximately $24 \mathrm{~h}$ (Liew and Kubes, 2019). In contrast, monocytes are long-lived immune cells that in monogastric species are known to polarize differently depending on their nutrient milieu (Linke et al., 2017; Wei et al., 2017). Bovine monocytes are a heterogeneous population and the $\mathrm{CD} 14^{+}$monocyte subset responds vigorously to pathogen-derived molecular patterns like LPS (Hussen et al., 2013; Hussen and Schuberth, 2017). We found that, contrary to PMN, monocytes showed marginal and inconsistent AKT activation in response to LPS stimulation relative to calving. In fact, AKT phosphorylation in monocytes numerically decreased below baseline postpartum rather than increasing, and was lower in all postpartum time points compared with 2 wk prepartum. This is consistent with our previous findings of decreased AKT activation in peripheral blood mononuclear cells postpartum (Mann et al., 2018). Postpartum depression of mTOR substrate responsiveness was only documented for S6RP, but S6RP generally showed a consistent response to LPS in monocytes. The reasons for our finding of robust AKT activation in PMN by LPS treatment, but general lack of activation in monocytes is unclear. Postpartum depression in responsiveness could indicate that nutrient sensing by mTORC1 plays a larger role in long-lived innate immune cells. This hypothesis is supported by our finding that enhancing the nutrient milieu in monocytes increases activation of the mTOR substrate 4EBP1 in the absence of LPS stimulation. Therefore, the supplementation with nutrients appears to preferentially target the mTORC1 substrate 4EBP1 compared with the S6 kinase pathway, which phosphorylates S6RP.

The second objective was to describe associations of these treatments with cytokine mRNA abundance profiles. The role of mTOR in human monocytes is described as regulatory and anti-inflammatory (Weichhart et al., 2008). The consistent nadir in kinase phosphorylation in the first week postpartum in mononuclear cells paired with an increase in phosphorylation in PMN could be indicative of a generally more pro-inflammatory response. This is consistent with the mRNA abundance data of whole blood leukocytes. Although subsets could not be investigated individually, inflammatory cytokine transcript production increased postpartum, particularly at baseline. This finding is similar to what we have reported previously in a larger cohort of transition cows (Mann et al., 2018). Addition of a nutrient mix consisting of AA, glucose, and insulin dampened the inflammatory response particularly for $I L 12 B$ and TNFA after stimulation. Few studies have evaluated cytokine changes in response to altering the nutrient milieu in bovine immune cells ex vivo or in vitro. Garcia et al. (2015) investigated the effect of glucose supplementation on PMN in vitro and found that when stimulated with $50 \mu \mathrm{g} / \mathrm{mL}$ LPS for $2 \mathrm{~h}$, glucose supplementation had no measurable effect on altering cytokine production. In a second study, the same group tested the effect of amino acid supplementation on PMN with the same stimulation protocol (Garcia et al., 2016). When supplementing the media with AA, mRNA expression of pro-inflammatory cytokines as well as media concentration of TNFa were decreased upon stimulation. Insulin was recently shown to alter pro-inflammatory or chemoattractant properties of central nervous macrophages (murine BV2 microglia cell line; Brabazon et al., 2018), but the effect of insulin on inflammatory cytokine production of bovine immune cells has not been investigated to the best of our knowledge. It is a limitation of our study that we cannot ascribe the observed effect to a single nutrient or insulin. Our goal was to supply a mixture of nutrients sensed and used by the mTOR pathway in this study. Future work should target the effect of individual nutrients (and individual AA) to attribute the anti-inflammatory effects to a certain nutrient or group of nutrients.

Cows in this study were enrolled to represent a typical cohort of cows undergoing transition from pregnancy to lactation. Based on the results of our markers of metabolic status, cows experienced a mild metabolic challenge during the transition period, however not to a level that would indicate a pronounced negative energy balance with subsequent higher risk of health disorders (Ospina et al., 2013). Studying cows that did not experience severe nutrient deficit at time of sampling may have affected changes due to artificial nutrient supplementation ex vivo. We hypothesize that responsiveness of mTOR pathway kinases to this nutrient supplementation may have been different if cows had been selected based on differences in metabolic status, milk production, or those experiencing health events.

The slight decrease in hematocrit as lactation progressed is consistent with previous observations and is thought to represent either a reduction of red blood cells or an increase in intravascular fluid volume (Wohlt et al., 1984). Changes in the composition of whole blood leukocyte populations over time are consistent with previous observations reported for healthy cows in the transition period (Moretti et al., 2016; McDougall et al., 2017). The most pronounced change over time was an increase in the percentage of PMN from $35 \%$ prepartum to $55 \%$ postpartum. Although our intracellular mTOR measurements were based on single cell measurements, the differences in gene expression as measured by mRNA abundance could have been af- 
fected by altered proportions of immune cell subsets, as well as by a change in immune cell cross-talk and activation. Therefore, in subsequent studies, immune cell subsets should be separated before nutrient supplementation and LPS stimulation. Although this will inhibit cross-talk and extract cells from their original milieu (whole blood), it will avoid confounding cytokine expression due to representation of different numbers of cell subsets typical of the bovine transition period.

The use of LPS as inflammatory signal in an ex vivo model was established with human whole blood and proved to be a valid tool to study immune modulatory effects in blood leukocytes (Wang et al., 2000). We used the ex vivo model to investigate the effect of different nutrient availability and inflammatory stimulation on the responsiveness of the nutrient sensing mTOR pathway in bovine blood leukocytes. It has to be noted that in this ex vivo model we added both nutrients and LPS in amounts that would be considered superphysiological under in vivo conditions. Determining the biological relevance of our ex vivo findings will require additional experimentation. The effect of nutrients on mTOR pathway activation in different leukocyte subsets could however be an indicator for nutrient manipulation as an immune modulatory tool in bovine $\mathrm{CD} 14^{+}$monocytes. Further investigations should consider mTOR pathway responsiveness in separated leukocyte subsets.

\section{CONCLUSIONS}

We showed that bovine innate immune cell types differ in their responsiveness of the mTOR pathway to LPS and differences in nutrient milieu ex vivo. In PMN, pathway activation was dominated by LPS effects and responsiveness was enhanced postpartum, whereas a postpartum depression in the responsiveness of the pathway was found for AKT and S6RP in monocytes. Responsiveness was different for pathway proteins with AKT showing response to LPS but limited response to nutrient supplementation, and 4EBP1 showing the greatest response to LPS when enhancing available nutrients. These results further confirm the differential role of pathway proteins in the integration of nutrient sensing and TLR-ligand response. Ex vivo mRNA abundance of inflammatory cytokines was altered in unstimulated and stimulated whole blood leukocytes when exposed to additional nutrients, shifting the overall response to a less pro-inflammatory phenotype. Our work confirms the role of mTOR signaling in LPS activated bovine immune cells, and the potential role of nutrient milieu on enhancing the responsiveness of downstream mTOR substrates. In a next step, nutrient supplementation and resulting inflammatory cytokine production need to be tested in individual cell popu- lations to determine which subsets alter their inflammatory response and phenotype in light of enhanced nutrient availability.

\section{ACKNOWLEDGMENTS}

This work was supported by the USDA grant number 2019-3610003618 administered by the Cornell University College of Veterinary Medicine (Ithaca, NY). Any opinions, findings, conclusions, or recommendations expressed in this publication are those of the author(s) and do not necessarily reflect the view of the National Institute of Food and Agriculture (NIFA) or the USDA. The authors thank Suzanne Klaessig (Cornell University, Ithaca, NY) for her invaluable assistance with data collection. We also thank Blake Nguyen (Cornell University, Ithaca, NY) and the Cornell University staff caring for the animals used in this study. The authors have not stated any conflicts of interest.

\section{REFERENCES}

Alvåsen, K., M. Jansson Mork, I. R. Dohoo, C. H. Sandgren, P. T. Thomsen, and U. Emanuelson. 2014. Risk factors associated with on-farm mortality in Swedish dairy cows. Prev. Vet. Med. 117:110 120. https://doi.org/10.1016/j.prevetmed.2014.08.011.

Bauerfeld, C. P., R. Rastogi, G. Pirockinaite, I. Lee, M. Huttemann, B. Monks, M. J. Birnbaum, L. Franchi, G. Nunez, and L. Samavati. 2012. TLR4-mediated AKT activation is MyD88/TRIF dependent and critical for induction of oxidative phosphorylation and mitochondrial transcription factor $\mathrm{A}$ in murine macrophages. J. Immunol. 188:2847-2857. https://doi.org/10.4049/jimmunol .1102157 .

Bionaz, M., E. Trevisi, L. Calamari, F. Librandi, A. Ferrari, and G. Bertoni. 2007. Plasma paraoxonase, health, inflammatory conditions, and liver function in transition dairy cows. J. Dairy Sci. 90:1740-1750. https://doi.org/10.3168/jds.2006-445.

Brabazon, F., S. Bermudez, M. Shaughness, G. Khayrullina, and K. R. Byrnes. 2018. The effects of insulin on the inflammatory activity of BV2 microglia. PLoS One 13:e0201878. https://doi.org/10.1371/ journal.pone.0201878.

Bradford, B. J., K. Yuan, J. K. Farney, L. K. Mamedova, and A. J. Carpenter. 2015. Invited review: Inflammation during the transition to lactation: New adventures with an old flame. J. Dairy Sci. 98:6631-6650. https://doi.org/10.3168/jds.2015-9683.

Brym, P., A. Rusc, and S. Kaminski. 2013. Evaluation of reference genes for qRT-PCR gene expression studies in whole blood samples from healthy and leukemia-virus infected cattle. Vet. Immunol. Immunopathol. 153:302-307. https://doi.org/10.1016/j .vetimm.2013.03.004

Cobbold, S. P. 2013. The mTOR pathway and integrating immune regulation. Immunology 140:391-398. https://doi.org/10.1111/ imm.12162.

Garcia, M., T. H. Elsasser, L. Juengst, Y. Qu, B. J. Bequette, and K. M. Moyes. 2016. Short communication: Amino acid supplementation and stage of lactation alter apparent utilization of nutrients by blood neutrophils from lactating dairy cows in vitro. J. Dairy Sci. 99:3777-3783. https://doi.org/10.3168/jds.2015-10086.

Garcia, M., T. H. Elsasser, Y. Qu, X. Zhu, and K. M. Moyes. 2015. Glucose supplementation has minimal effects on blood neutrophil function and gene expression in vitro. J. Dairy Sci. 98:6139-6150. https://doi.org/10.3168/jds.2014-9183.

Haidinger, M., M. Poglitsch, R. Geyeregger, S. Kasturi, M. Zeyda, G. J. Zlabinger, B. Pulendran, W. H. Horl, M. D. Saemann, and T. 
Weichhart. 2010. A versatile role of mammalian target of rapamycin in human dendritic cell function and differentiation. J. Immunol. 185:3919-3931. https://doi.org/10.4049/jimmunol.1000296.

Hammon, D. S., I. M. Evjen, T. R. Dhiman, J. P. Goff, and J. L. Walters. 2006. Neutrophil function and energy status in Holstein cows with uterine health disorders. Vet. Immunol. Immunopathol. 113:21-29. https://doi.org/10.1016/j.vetimm.2006.03.022.

Hussen, J., A. Düvel, O. Sandra, D. Smith, I. M. Sheldon, P. Zieger, and H.-J. Schuberth. 2013. Phenotypic and functional heterogeneity of bovine blood monocytes. PLoS One 8:e71502. https://doi .org/10.1371/journal.pone.0071502.

Hussen, J., and H. J. Schuberth. 2017. Heterogeneity of bovine peripheral blood monocytes. Front. Immunol. 8:1875. https://doi.org/10 .3389/fimmu.2017.01875.

Jones, R. G., and E. J. Pearce. 2017. MenTORing Immunity: mTOR signaling in the development and function of tissue-resident immune cells. Immunity 46:730-742. https://doi.org/10.1016/j .immuni.2017.04.028.

Katholnig, K., M. Linke, H. Pham, M. Hengstschlager, and T. Weichhart. 2013. Immune responses of macrophages and dendritic cells regulated by mTOR signalling. Biochem. Soc. Trans. 41:927-933. https://doi.org/10.1042/BST20130032.

Kimura, K., J. P. Goff, M. E. Kehrli Jr., and J. A. Harp. 1999. Phenotype analysis of peripheral blood mononuclear cells in periparturient dairy cows. J. Dairy Sci. 82:315-319. https://doi.org/10.3168/ jds.S0022-0302(99)75238-0.

Laplante, M., and D. M. Sabatini. 2009. mTOR signaling at a glance. J. Cell Sci. 122:3589-3594. https://doi.org/10.1242/jcs.051011.

Leal Yepes, F. A., D. V. Nydam, W. Heuwieser, and S. Mann. 2018. Technical note: Evaluation of the diagnostic accuracy of 2 pointof-care beta-hydroxybutyrate devices in stored bovine plasma at room temperature and at 37 degrees C. J. Dairy Sci. 101:64556461. https://doi.org/10.3168/jds.2017-13960.

Liew, P. X., and P. Kubes. 2019. The neutrophil's role during health and disease. Physiol. Rev. 99:1223-1248. https://doi.org/10.1152/ physrev.00012.2018.

Linke, M., S. D. Fritsch, N. Sukhbaatar, M. Hengstschläger, and T. Weichhart. 2017. mTORC1 and mTORC2 as regulators of cell metabolism in immunity. FEBS Lett. 591:3089-3103. https://doi .org/10.1002/1873-3468.12711.

Mann, S., A. Abuelo, D. V. Nydam, F. A. Leal Yepes, T. R. Overton, and J. J. Wakshlag. 2016b. Insulin signaling and skeletal muscle atrophy and autophagy in transition dairy cows either overfed energy or fed a controlled energy diet prepartum. J. Comp. Physiol. B 186:513-525. https://doi.org/10.1007/s00360-016-0969-1.

Mann, S., F. A. L. Yepes, E. Behling-Kelly, and J. A. A. McArt. 2017. The effect of different treatments for early-lactation hyperketonemia on blood beta-hydroxybutyrate, plasma nonesterified fatty acids, glucose, insulin, and glucagon in dairy cattle. J. Dairy Sci. 100:6470-6482. https://doi.org/10.3168/jds.2016-12532.

Mann, S., D. V. Nydam, A. Abuelo, F. A. Leal Yepes, T. R. Overton, and J. J. Wakshlag. 2016a. Insulin signaling, inflammation, and lipolysis in subcutaneous adipose tissue of transition dairy cows either overfed energy during the prepartum period or fed a controlled-energy diet. J. Dairy Sci. 99:6737-6752. https://doi.org/10 $.3168 /$ jds.2016-10969.

Mann, S., A. Sipka, F. A. Leal Yepes, D. V. Nydam, T. R. Overton, and J. J. Wakshlag. 2018. Nutrient-sensing kinase signaling in bovine immune cells is altered during the postpartum nutrient deficit: A possible role in transition cow inflammatory response. J. Dairy Sci. 101:9360-9370. https://doi.org/10.3168/jds.2018-14549.

McDougall, S., S. J. LeBlanc, and A. Heiser. 2017. Effect of prepartum energy balance on neutrophil function following pegbovigrastim treatment in periparturient cows. J. Dairy Sci. 100:7478-7492. https://doi.org/10.3168/jds.2017-12786.

Medina, E. A., I. R. Morris, and M. T. Berton. 2010. Phosphatidylinositol 3-kinase activation attenuates the TLR2-mediated macro- phage proinflammatory cytokine response to Francisella tularensis live vaccine strain. J. Immunol. 185:7562-7572. https://doi.org/10 .4049/jimmunol.0903790.

Moretti, P., M. Probo, A. Cantoni, S. Paltrinieri, and A. Giordano. 2016. Fluctuation of neutrophil counts around parturition in Holstein dairy cows with and without retained placenta. Res. Vet. Sci. 107:207-212. https://doi.org/10.1016/j.rvsc.2016.06.015.

Ospina, P. A., J. A. McArt, T. R. Overton, T. Stokol, and D. V. Nydam. 2013. Using nonesterified fatty acids and beta-hydroxybutyrate concentrations during the transition period for herd-level monitoring of increased risk of disease and decreased reproductive and milking performance. Vet. Clin. North Am. Food Anim. Pract. 29:387-412. https://doi.org/10.1016/j.cvfa.2013.04.003.

Shahid, M. Q., J. K. Reneau, H. Chester-Jones, R. C. Chebel, and M. I. Endres. 2015. Cow- and herd-level risk factors for on-farm mortality in Midwest US dairy herds. J. Dairy Sci. 98:4401-4413. https://doi.org/10.3168/jds.2014-8513.

Sordillo, L. M., G. M. Pighetti, and M. R. Davis. 1995. Enhanced production of bovine tumor necrosis factor- $\alpha$ during the periparturient period. Vet. Immunol. Immunopathol. 49:263-270. https:/ /doi.org/10.1016/0165-2427(95)05465-0 http://dx.doi.org/http:// dx.doi.org/10.1016/0165-2427(95)05465-0.

Troutman, T. D., J. F. Bazan, and C. Pasare. 2012. Toll-like receptors, signaling adapters and regulation of the pro-inflammatory response by PI3K. Cell Cycle 11:3559-3567. https://doi.org/10 $.4161 /$ cc. 21572 .

Vergadi, E., E. Ieronymaki, K. Lyroni, K. Vaporidi, and C. Tsatsanis. 2017. Akt signaling pathway in macrophage activation and M1/ M2 polarization. J. Immunol. 198:1006-1014. https://doi.org/10 .4049/jimmunol.1601515.

Wang, J. E., R. Solberg, C. Okkenhaug, P. F. Jorgensen, C. D. Krohn, and A. O. Aasen. 2000. Cytokine modulation in experimental endotoxemia: Characterization of an ex vivo whole blood model. Eur. Surg. Res. 32:65-73. https://doi.org/10.1159/000008743.

Wei, J., J. Raynor, T.-L. M. Nguyen, and H. Chi. 2017. Nutrient and metabolic sensing in T cell responses. Front. Immunol. 8:247. https://doi.org/10.3389/fimmu.2017.00247.

Weichhart, T., G. Costantino, M. Poglitsch, M. Rosner, M. Zeyda, K. M. Stuhlmeier, T. Kolbe, T. M. Stulnig, W. H. Horl, M. Hengstschlager, M. Muller, and M. D. Saemann. 2008. The TSC-mTOR signaling pathway regulates the innate inflammatory response. Immunity 29:565-577. https://doi.org/10.1016/j.immuni.2008.08 .012 .

Weichhart, T., M. Hengstschlager, and M. Linke. 2015. Regulation of innate immune cell function by mTOR. Nat. Rev. Immunol. 15:599-614. https://doi.org/10.1038/nri3901.

Wohlt, J. E., J. L. Evans, and J. R. Trout. 1984. Blood constituents in lactating Holstein cows influenced by hematocrit, sampling site, and diet protein and calcium. J. Dairy Sci. 67:2236-2246. https:// doi.org/10.3168/jds.S0022-0302(84)81571-4.

Zhao, M., C. Li, F. Shen, M. Wang, N. Jia, and C. Wang. 2017. Naringenin ameliorates LPS-induced acute lung injury through its anti-oxidative and anti-inflammatory activity and by inhibition of the PI3K/AKT pathway. Exp. Ther. Med. 14:2228-2234. https:// doi.org/10.3892/etm.2017.4772.

\section{ORCIDS}

Anja S. Sipka @ https://orcid.org/0000-0002-8646-824X

Tawny L. Chandler () https://orcid.org/0000-0001-6299-9436

Erica L. Behling-Kelly @i https://orcid.org/0000-0001-9189-4238

Thomas R. Overton @ https://orcid.org/0000-0003-4098-4916

Sabine Mann ำ https://orcid.org/0000-0003-1806-1154 